\title{
MIXING LAYERS IN FLOWS IN STAR FORMING REGIONS
}

\author{
A. C. RAGA AND J. CANTÓ \\ Instituto de Astronomía, UNAM, Ap. 70-264 \\ 04510 México, D. F., México
}

\begin{abstract}
There are now many observations of high velocity, molecular emission associated with outflows from young stars. This emission might come from molecules that are formed in the outflowing material, or from entrained, ambient molecular gas. The present paper explores the latter possibility, describing the efforts that have been made to model the "lateral entrainment" that occurs in the turbulent mixing layer formed along the edges of a jet-like flow (or, equivalently, at any interface between a fast moving flow and the surrounding environment). A simple, analytic approach based on model equations is used to provide a qualitative picture of the dynamical, thermal and chemical structure of such a turbulent mixing layer. Finally, a review of the efforts up to date of modelling the dynamics and chemistry of turbulent mixing layers is presented.
\end{abstract}

\section{Introduction}

Bipolar outflows from young stars are observed in the UV, optical and IR lines of atoms and ions (with a range of different ionization states), in the radio, free-free continuum and in UV, IR and radio lines of different molecules. It has generally been observed that the molecular emission in the so-called "molecular outflows" has radial velocities in the $10-30 \mathrm{~km} / \mathrm{s}$ range. On the other hand, the radial velocities and proper motions of the atomic/ionic Herbig-Haro (HH) objects and/or jets indicate velocities typically in the $50-500 \mathrm{~km} / \mathrm{s}$ range. These results have been reviewed, e.g., by Reipurth \& Cernicharo (1995) and by Böhm (1995).

However, recent high-spatial resolution radio $\mathrm{CO}$ observations have revealed that the on-axis regions of at least some molecular outflows have $\sim 100 \mathrm{~km} / \mathrm{s}$ radial velocities, which are comparable to the velocities of the atomic/ionic component (see, e.g., Richer, Hills \& Padman 1992 and 
the review of Cabrit 1995). IR observations of $\mathrm{H}_{2}$ emission also show that molecular gas with velocities of more than $100 \mathrm{~km} / \mathrm{s}$ is present (Zinnecker et al. 1989).

It is of course not clear how this high velocity molecular gas is formed. There are two distinct possibilities:

$i$. the molecules are either formed or already present in the outflowing material,

$i i$. the molecules trace environmental material that has been entrained into the high velocity outflow.

The first possibility has been explored by Rawlings, Williams \& Cantó (1988) and by Glassgold, Mamon \& Huggins (1989), who find that it is only possible to form molecules in a wind from a $\mathrm{T}$ Tauri star if the associated mass loss rate has values of $\dot{M}>10^{-6} \mathrm{M}_{\odot} \mathrm{yr}^{-1}$, which might be somewhat high at least for some outflows from young stars.

The present review is concerned with the second scenario, in which environmental, molecular gas is entrained into an atomic/ionic outflow. The problem to be solved in this scenario is the nature of the mechanisms by which molecular material can be entrained and accelerated to velocities comparable to the outflow velocity without dissociating the molecules present in the environmental gas.

The processes of entrainment in a jet flow are usually divided into the socalled "lateral entrainment" (which takes place in a turbulent mixing layer formed along the lateral interface between the jet beam and the surrounding environment) and "head entrainment" (a term that is used to describe the pushing and mixing that takes place at the head of the jet and its associated vortices). The spatial location of these two mechanisms is shown in Figure 1.

The present review is concerned with the turbulent mixing layers relevant for "lateral entrainment" (see Figure 1). Sections 2-5 describe a simple, analytic model (based on a "turbulent viscosity" approach) describing the cross section of a mixing layer. Section 6 summarizes the results obtained in the past from mixing layer models. Finally, section 7 discusses possible directions in which this field might evolve during the next few years.

\section{The dynamical structure of turbulent mixing layers}

If we have two interacting, high Reynolds number flows moving at different velocities, the region of interaction has the structure shown in Figure 2. A standoff shock can be generated in one or in both of the flows (shocks S1 and S2 of Figure 2), and a turbulent mixing layer is formed at the interface between the two flows (as a result of the nonlinear development of Kelvin-Helmholtz instabilities at the boundary). 


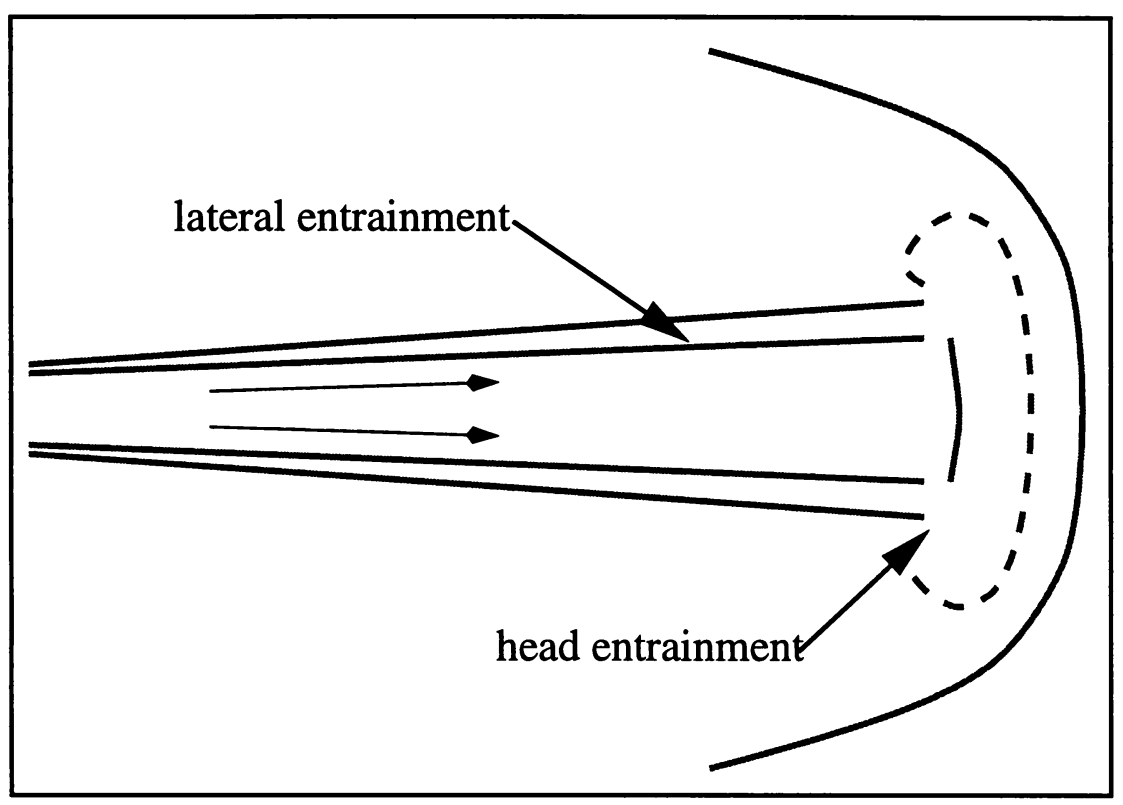

Figure 1. Schematic diagram of a jet flow, showing the location of the lateral and head entrainment regions

Let us consider the most simple, possible case: a plane, supersonic beam (which we will call the "jet") which is in pressure equilibrium with the surrounding environment. In this case, no standoff shocks are generated, and the resulting situation is as illustrated in Figure 3.

From laboratory experiments in the $M_{j}=1-20$ Mach number range (Harvey \& Hunter 1975; Birch \& Eggers 1972) it is known that the mean flow in the mixing layer is roughly parallel to the direction of the undisturbed flow. The equation of motion for the mean flow can then be written as:

$$
\mu \frac{d^{2} v}{d y^{2}}=0
$$

where $\mu$ is the turbulent viscosity, $v$ is the component of the mean flow along the mixing layer and $y$ is the coordinate measured from the jet into the mixing layer (see Figure 3). This equation can be straightforwardly integrated to obtain:

$$
v(\eta)=v_{j}(1-\eta)
$$




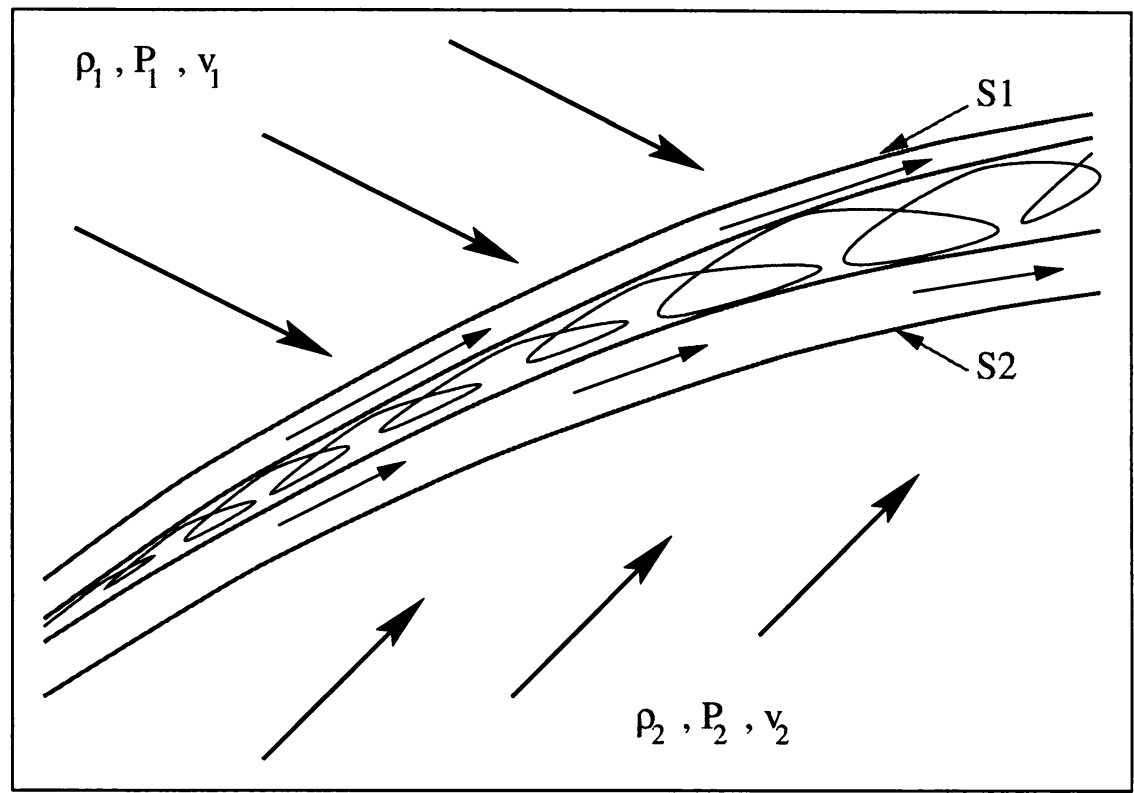

Figure 2. Schematic diagram of the general configuration of the interface between two flows. Two shocks (S1 and S2) and a turbulent mixing layer are formed.

with $\eta=y / h$, where $h$ is the width of the mixing layer (see Figure 3). This is of course the well known Couette flow solution. It is found that laboratory mixing layers do have an almost linear mean flow cross section (except for edge effects at $y \approx 0$ and $y \approx h$ ) in agreement with equation (2). Superimposed on this mean flow there are of course time-dependent turbulent motions, which have a typical velocity $\ll v_{j}$.

\section{Thermal structure}

The energy equation describing the temperature cross section of a turbulent mixing layer can be written as a balance of turbulent conduction, turbulent dissipation and radiative energy loss:

$$
\kappa \frac{d^{2} T}{d y^{2}}+\mu\left(\frac{d v}{d y}\right)^{2}=L
$$

where $L$ is the radiative energy loss (per unit volume) and $\kappa$ is the turbulent conductivity, which we assume to be independent of $y$. Using the experimental result that the turbulent Prandtl number is $\operatorname{Pr}=\mu c_{p} / \kappa \approx 1$, we can write equation (3) as: 


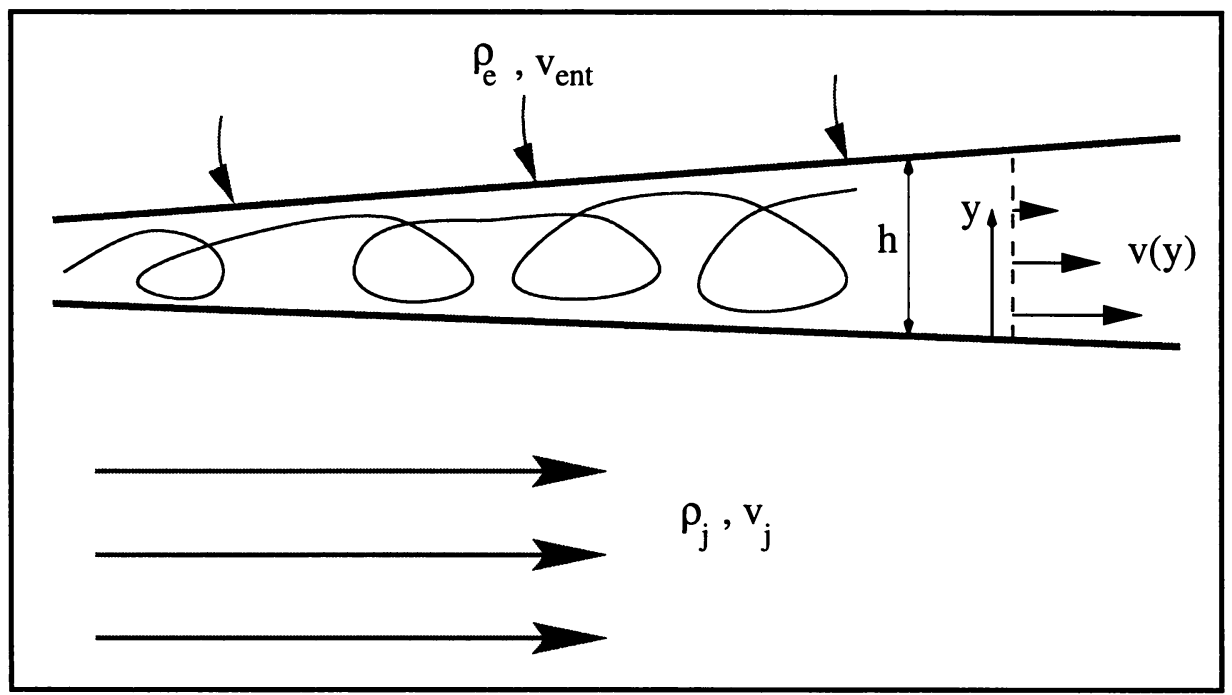

Figure 3. Schematic diagram of the mixing layer formed between a supersonic, pressure matched jet and a stationary environment. The environmental material is dragged into the mixing layer at a highly subsonic "entrainment velocity" $v_{e n t}$.

$$
\frac{d^{2} t}{d \eta^{2}}+M_{0}^{2}=l
$$

where $\eta=y / h, t=T / T_{0}, M_{0}^{2}=(\gamma-1) m v_{j}^{2} /\left(\gamma k T_{0}\right)$ and $l=(\gamma-1)$ $m h^{2} L /\left(\gamma k T_{0} \mu\right)$. We have adimensionalized $T$ with a temperature $T_{0}$ which we take to be $T_{0} \approx 12000 \mathrm{~K}$, at which the cooling function $L$ has a steep increase with temperature.

In order to proceed analytically, we consider the idealized dimensionless cooling function:

$$
\begin{gathered}
l(\rho, t)=0 ; t \leq 1, \\
l(\rho, t)=\infty ; t>1 .
\end{gathered}
$$

With this cooling function and the boundary conditions $t(\eta=0)=t_{j}=$ $T_{j} / T_{0}$ and $t(\eta=1)=t_{e}=T_{e} / T_{0}$, equation (4) can be integrated to obtain:

$$
\begin{gathered}
t(\eta)=\frac{M_{0}^{2}}{2} \eta(1-\eta)+t_{e} \eta+t_{j}(1-\eta), \\
\text { for } \quad M_{0} \leq M_{0, c}=\sqrt{2\left(1-t_{j}\right)}+\sqrt{2\left(1-t_{e}\right)} .
\end{gathered}
$$


In other words, for $M_{0} \leq M_{0, c}$, we have $t(\eta) \leq 1$, and therefore we regain the well known parabolic temperature cross-section of an adiabatic turbulent mixing layer. For $M_{0}>M_{0, c}$ (see equation 7), the solution of equation (4) takes the form:

$$
\begin{gathered}
t(\eta)=M_{0}^{2}\left[\eta_{1} \eta-\frac{\eta^{2}}{2}\right]+t_{j} ; \quad 0 \leq \eta<\eta_{1}, \\
t(\eta)=1 ; \quad \eta_{1} \leq \eta \leq \eta_{2} \\
t(\eta)=M_{0}^{2}\left[\left(1-\eta_{2}\right)(1-\eta)-\frac{(1-\eta)^{2}}{2}\right]+t_{e} ; \quad \eta_{2}<\eta \leq 1
\end{gathered}
$$

where

$$
\begin{gathered}
\eta_{1}=\frac{1}{M_{0}} \sqrt{2\left(1-t_{j}\right)}, \\
\eta_{2}=1-\frac{1}{M_{0}} \sqrt{2\left(1-t_{e}\right)},
\end{gathered}
$$

This solution (equations 8 and 9) has a flat region for $\eta_{1} \leq \eta \leq \eta_{2}$, and quadratic "wings" at both edges of the mixing layer cross section, as shown in Figure 4. For $M_{0} \gg 1$, the isothermal plateau ranges from $\eta_{1} \approx 0$ to $\eta_{2} \approx 1$ (see equation 9 ), so that basically the whole cross section is isothermal, with $t=1$ (or, in dimensional form, $T=T_{0}$ ).

If the mixing layer is narrow, the material in the cross section will be in pressure balance with the jet and the environment (which are assumed to be pressure matched, see above). Because of this, if the average mass per particle $m$ is independent of $y$, the density will also be uniform across the mixing layer (except of course at the edges, where the temperature deviates from $T_{0}$ ).

\section{The molecular/atomic/ionic structure}

Let us consider the fraction $f$ of atomic nuclei that are in a specified atomic, ionic or molecular state. This fractional abundance obeys the diffusion equation:

$$
D \frac{d^{2} f}{d y^{2}}=-S_{f}
$$

where we have assumed that the turbulent diffusion coefficient $D$ is independent of $y . S_{f}$ is the rate of production (or destruction if $S_{f}<0$ ) of the 


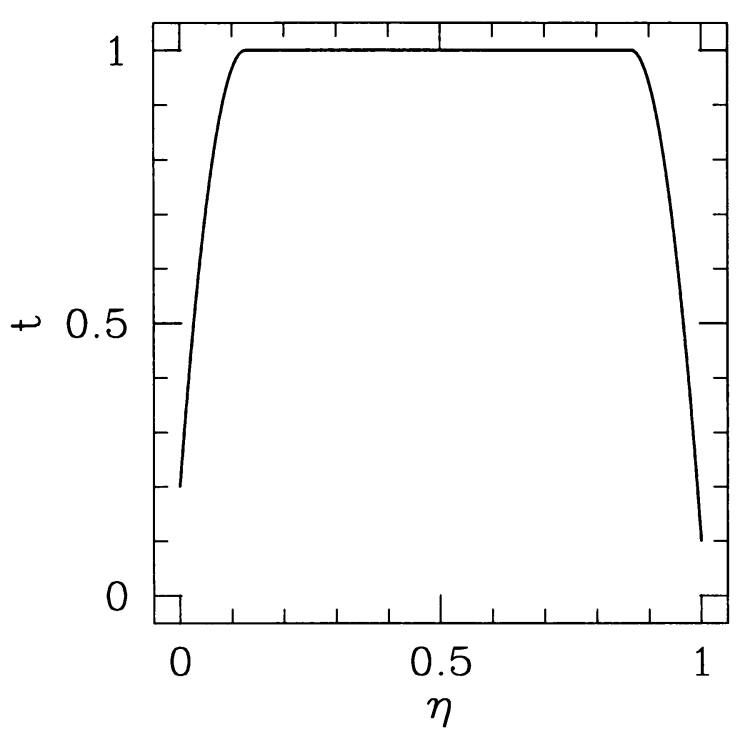

Figure 4. Temperature cross section $t(\eta)$ (where $t=T / T_{0}$ is the dimensionless temperature, and $\eta=y / h$ the dimensional position across the mixing layer, see the text) for a mixing layer with $M_{0}=10$, formed between a jet with dimensionless temperature $t_{j}=0.2$ and an environment with $t_{e}=0.1$.

species under consideration. This term couples equation (10) to a system of similar equations for all of the relevant species.

In order to proceed analytically, we write equation (10) in dimensionless form:

$$
\frac{d^{2} f}{d \eta^{2}}=-s_{f}
$$

where $\eta=y / h$ and $s_{f}=h^{2} S_{f} / D$, and we then consider the simple "destruction" source term $s_{f}=-\sigma f$ with constant $\sigma$. This source term would correspond to a species which is destroyed by interactions with a reactant which has an abundance that does not depend on $y$, in an isothermal mixing layer (see the previous section). With this simple, model source term, equation (11) can be integrated to obtain:

$$
f(\eta)=\frac{f_{e} \sinh [\sigma \eta]+f_{j} \sinh [\sigma(1-\eta)]}{\sinh \sigma},
$$

where $f_{j}$ and $f_{e}$ are the values of $f$ for the jet and for the environment (respectively). 


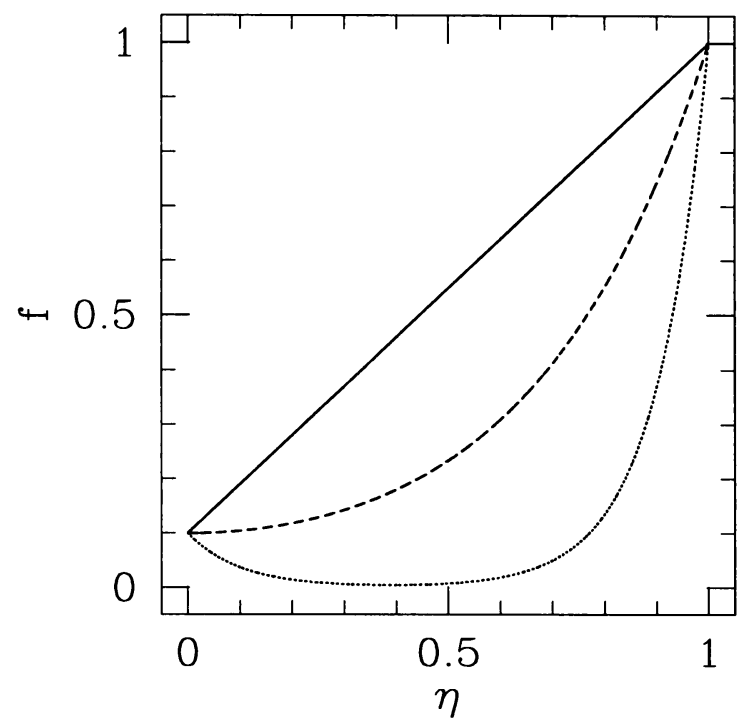

Figure 5. The fractional abundance $f$ (of a chemical species) as a function of the dimensionless position $\eta=y / h$ across the mixing layer, obtained from equation 12 (see the text). We have assumed that the jet material has an abundance $f_{j}=0.1$ (at $\eta=0$ ), and that the environment has $f_{e}=1($ at $\eta=1)$. The figures show the results obtained for $\sigma=0.1$ (solid line), $\sigma=3$ (dashed line) and $\sigma=10$ (dotted line).

For $\sigma \ll 1$, equation (12) gives a straight line joining $\eta=0, f=f_{j}$ with the point $\eta=1, f=f_{e}$ (i.e., the standard solution to the source-less diffusion equation in plane geometry). This is the limit of a "chemically inactive" mixing layer, in which the abundance of the different species is fixed by a weighted mixture of the jet and environmental material. From the definition of the dimensionless source function $s_{f}$ (see the discussion following equation 11), it is clear that a very narrow mixing layer is always in this regime.

For $\sigma \sim 1$, equation (12) starts to develop a dip in the central region of the mixing layer cross section, and for $\sigma \gg 1$, it gives $f \approx 0$ except at the edges of the cross section. These "chemically active" solutions are plotted in Figure 5.

\section{The values of the transport coefficients}

The remaining problem is to determine the values of the transport coefficients $\mu, \kappa$ and $D$ (see equations 3 and 10). Through a fit to hypersonic mixing layer experiments, Cantó \& Raga (1991) derived a "mixing length" 
prescription for the turbulent viscosity:

$$
\mu=\alpha \bar{\rho} \bar{c} h,
$$

where $\alpha \approx 0.007$, and $\bar{\rho}$ and $\bar{c}$ are the density and sound speed (respectively) averaged across the width of the mixing layer.

An important problem with this prescription is that there is no clear way of calculating $\bar{\rho}$ and $\bar{c}$. For example, these could be computed as unweighted averages across the width of the mixing layer, but there is no clear reason for this to be so. However, for the case of a high Mach number, quasiisothermal mixing layer (see the results from section 3), both $\rho$ and $c$ are almost constant, so that the problem of how to compute $\bar{\rho}$ and $\bar{c}$ becomes less important.

Once $\mu$ has been computed (e.g., with equation 13), the two remaining transport coefficients (see equations 3 and 10) can be calculated as $\kappa=\mu / c_{p}$ and $D=\mu / \bar{\rho}$. These simple prescriptions for $\kappa$ and $D$ derive from the experimental result that the Prandtl number and the diffusion Prandtl number of turbulent transport have values $\approx 1$.

\section{Mixing layer calculations}

In sections 2-5 we have derived a simplified version of the equations necessary for describing a mixing layer, based on a "turbulent viscosity" parametrization of the turbulent motions. As these equations are second order, diffusion-type differential equations, they are somewhat more difficult to solve than the ordinary differential equations describing, e.g., the cooling region behind a shock wave. Possibly because of this reason, only very few calculations with a treatment of the associated atomic/chemical network have been carried out.

In the following, we will summarize the calculations that have been made in the past, dividing them into seven different categories:

$i$. General properties of mixing layers

Kahn (1980) presented a detailed description of linear instabilities in a shear layer, and their nonlinear development. Hartquist \& Dyson (1988) discuss the dynamical properties of mixing layers from a more physical (and less mathematically complex) point of view. Stahler (1994) discusses the properties of mixing layers from a mostly qualitative way.

ii. Single parcel, dynamical models

A dynamical, single parcel model (based on quantities averaged across the width of the mixing layer) was presented by Cantó \& Raga (1991), who carried out comparisons with results from laboratory experiments. Raga, Cabrit \& Cantó (1995) extended these models to consider the whole system of curved standoff shocks+turbulent mixing layer (see 
Figure 2). Raga et al. (1993) applied similar ideas to the case of a fully turbulent, stellar jet.

iii. Single parcel, chemical models

Charnley et al. (1990), Williams \& Hartquist (1991) and Nejad \& Hartquist (1994) considered chemical models of a single parcel into which material of different chemical composition is injected (in order to simulate the effect of entrainment). These models give predictions of the chemical species that would preferentially be produced in mixing layers.

$i v$. Single parcel, dynamical models with chemistry

Taylor \& Raga (1995) extended the dynamical model of Cantó \& Raga (1991) to include a chemical network similar to the one of Nejad \& Hartquist (1994).

v. Spatially resolved, dynamical models

Noriega-Crespo et al. (1996) describe a simplified mixing layer model based on a turbulent viscosity parametrization (the equations described in sections 2-5 are based on this paper). Malone, Dyson \& Hartquist (1994) derive an alternative mixing layer model, based on a parametrization of the mass and momentum deposition rates from the "jet" onto the environmental material.

vi. Spatially resolved, chemical models

Chièze et al. (1991) and Xie et al. (1995) compute models of the effect of turbulent transport on the chemistry of the gas, but apply these models to full, turbulent clouds (rather than to mixing layers). Rawlings \& Hartquist (1996) compute models of a diffusive mixing layer, with a linear $T(y)$ cross section.

vii. Spatially resolved, dynamical models with chemistry

Dyson et al. (1995) have computed models including a simplified chemical network in their dynamical mixing layer formalism (Malone et al. 1994). Giovanardi \& Lizano (1995) and Lizano \& Giovanardi (1995) presented a dynamical+chemical model based on an entirely different approach, in which the flow field itself is parametrized so as to match observations of molecular outflows.

\section{Discussion: possible ways of advancing in this field}

As it is clear from the previous section, the work that has been done in the past concerning turbulent mixing layers is at best somewhat sketchy. This is partly a result of the fact that there is no clear theoretical framework for treating turbulent flows, and partly a result of the higher than usual degree of complexity of even the most simple model equations.

For engineering applications, the traditional way of modelling turbulent flows (and, in particular, boundary layers) has been to parametrize 
the effect of the turbulent eddies by a "turbulent" (or "eddie") viscosity, as pioneered by some of the "parents" of the field of gas dynamics (e.g., Prandtl). The calculation of this viscosity ranges from straightforward, mixing length recipes (see, e.g., section 5) to the so-called "second order" (or "third order") closure methods. In these latter methods, a semi-empirical differential equation is used to describe the transport, e.g., of the turbulent energy, and the turbulent viscosity is then calculated as a function of this energy. A method of this kind is the so-called "K- $\epsilon$ " turbulence model (Dash \& Wolf 1983).

The main problem of such parametrizations of the turbulence is that they are normally calibrated with laboratory flows of Mach numbers $M \sim 0$ 5. Even though the parametrizations work astoundingly well within this Mach number range (as evidenced by the fact that the prototypes of newly designed planes are able to take off on their first flight), it is unclear whether or not they work properly for the $M \sim 10-30$ range, which is more relevant for flows in star formation regions.

There are at least two parametrizations that do not appear to suffer from this problem:

$i$. the mixing length parametrization of Cantó \& Raga (1991), which has been calibrated with experiments of up to $M \sim 20$,

$i i$. the K- $\epsilon$ parametrization, which has been modified by Falle (1994, following the suggestions of Zeman 1990), and calibrated with a $M=9$ flow experiment.

While the first of these "turbulence models" is more simple, and better suited for one-dimensional mixing layer models with extended chemistry, the second model is probably more accurate, and also better suited for full gasdynamic simulations of turbulent flows.

There are also two other independent approaches to modelling turbulent flows, which as far as we are aware are indigenous to astrophysics:

iii. the "mass and momentum injection" parametrization of Malone et al. (1994),

$i v$. the "parametrized flow" model of Lizano \& Giovanardi (1995).

These two approaches (described in the previous section) are interesting because they result in first order differential equations (rather than the higher order equations of turbulent viscosity models). However, at least as far as we are aware, no attempt has been made to compare these parametrizations with laboratory experiments, which appears to be a required step for progress to be made in any semi-empirical turbulence model.

On the other hand, it is not completely clear that calibrations of turbulence parametrizations with laboratory experiments are directly applicable to models of flows in star formation regions. There are published results 
from laboratory experiments of flows with Mach numbers $M \approx 20$, and in the so-called "high Reynolds number limit", so that both $M$ and $R e$ are indeed in the relevant parameter range.

However, these experiments all correspond to adiabatic flows, in which the Mach number in the turbulent regions is lowered to $M \sim 1-2$, as a result of the strong turbulent heating. This is clearly different in stellar outflows, where the strong radiative cooling keeps most of the turbulent regions at a temperature $T \sim 10^{4} \mathrm{~K}$, so that their Mach number can have values of $M>10$. This qualitative difference between stellar outflows and laboratory experiments clearly raises questions about the astrophysical use of laboratory calibrated turbulence parametrizations.

We believe that this problem should not stop us from developing detailed models of astrophysical mixing layers (including both the dynamical and chemical aspects), and that such models should be calculated in the near future. Regardless of the precision of the turbulence parametrization used, these models would be most helpful to illucidate the mechanisms by which molecular material can be accelerated to the high velocities observed in star formation regions.

As a comparison, we have the work done on convection in stellar atmospheres and interiors. In this field, a considerable amount of progress has been made using a mixing length model that has much less experimental backing than some of the parametrizations described in the present paper.

Very substantial progress could be made with laboratory experiments of non-adiabatic, hypersonic mixing layers. Though it is clear that in the laboratory it would be impossible to have a flow with a short enough cooling distance for radiative energy losses to be important, it might be possible to simulate such effects by introducing droplets of an appropriate liquid into the flow. For well chosen flow parameters, the latent heat of evaporation of the liquid would remove thermal energy from the gas, resulting in a dynamical effect similar to the one of a strong radiative cooling.

Direct numerical simulations might provide some insight into the problem of turbulent mixing in a hypersonic, radiative flow. However, the resolution that can be attained in such simulations limits them to the case of relatively low $(\leq 1000)$ Reynolds number flows, a regime that is inappropriate for flows in star formation regions. Given this, together with the fact that a complete theory of turbulent motions is unlikely to be derived in the near future, appropriate laboratory experiments might provide the most promising way forward for obtaining a better understanding of hypersonic, radiative mixing layers. 


\section{Acknowledgements}

We acknowledge support from the CONACYT grant number 0177P-E.

\section{References}

Birch, S. F., and Eggers, J. M. 1972, in Free Turbulent Shear Flows, Vol. I, Conf. Proc. NASA SP-321, p. 11.

Böhm, K. H. 1995, Ap\&SS 233, 11.

Cabrit, S. 1995, Ap\&SS 233, 81.

Cantó, J., and Raga, A. C. 1991, ApJ 372, 626.

Charnley, S. B., Dyson, J. E., Hartquist, T. W., and Williams, D. A. 1990, MNRAS 243, 405.

Chièze, J. P., Pineau des Forêts, G., and Herbst, E. 1991, ApJ, 373, 110.

Dash, S. M., and Wolf, D. E. 1983, AIAA paper 83-0704.

Dyson, J. E., Hartquist, T. W., Malone, M. T., and Taylor, S. D. 1995, in Circumstellar Disks, Outflows and Star Formation, eds. S. Lizano and J. M. Torrelles, RevMexAA Conf. Series, 1, p. 119.

Falle, S. A. E. G. 1994, MNRAS, 269, 607.

Giovanardi, C., and Lizano, S. 1995, in Circumstellar Disks, Outflows and Star Formation, eds. S. Lizano and J. M. Torrelles, RevMexAA Conf. Series, 1, p. 129.

Glassgold, A. E., Mamon, G. A., and Huggins, P. J. 1989, ApJ, 336, L29.

Hartquist, T. W., and Dyson, J. E. 1988, Ap\& SS, 144, 615.

Harvey, W. D., and Hunter, W. W. 1975, NASA Tech. Note D-7981.

Kahn, F. D. 1980, A\&A, 83, 303.

Lizano, S., and Giovanardi, C. 1995, ApJ, 447, 742.

Malone, M. T., Dyson, J. E., and Hartquist, T. W. 1994, Ap\&SS, 216, 143.

Nejad, L. A. M., and Hartquist, T. W. 1994, Ap\&SS, 220, 253.

Noriega-Crespo, A., Garnavich, P. M., Raga, A. C., Cantó, J., and Böhm, K. H. 1996, ApJ, 462, 804.

Raga, A. C., Cabrit, S., and Cantó, J. 1995, MNRAS, 273, 422.

Raga, A. C., Cantó, J., Calvet, N., Rodríguez, L. F., and Torrelles, J. M. 1993, A\&A, 276,539 .

Rawlings, J. M. C., and Hartquist, T. W. 1996, ApJ (submitted).

Rawlings, J. M. C., Williams, D. A., and Cantó, J. 1988, MNRAS, 230, 695.

Reipurth, B., and Cernicharo, J. 1995, in Circumstellar Disks, Outflows and Star Formation, eds. S. Lizano and J. M. Torrelles, RevMexAA Conf. Series, 1, p. 43.

Richer, J. S., Hills, R. E., and Padman, R. 1992, MNRAS, 254, 525.

Stahler, S. 1994, ApJ, 422, 616.

Taylor, S. D., and Raga, A. C. 1995, A\&A, 296, 823.

Williams, D. A., and Hartquist, T. W. 1991, MNRAS, 251, 351.

Xie, T., Allen, M., and Langer, W. D. 1995, ApJ, 440, 674.

Zeman, O. 1990, Phys. Fluids, A2, 178.

Zinnecker, H., Mundt, A., Geballe, T. R. and Zealy, W. J. 1989, ApJ, 342, 337. 


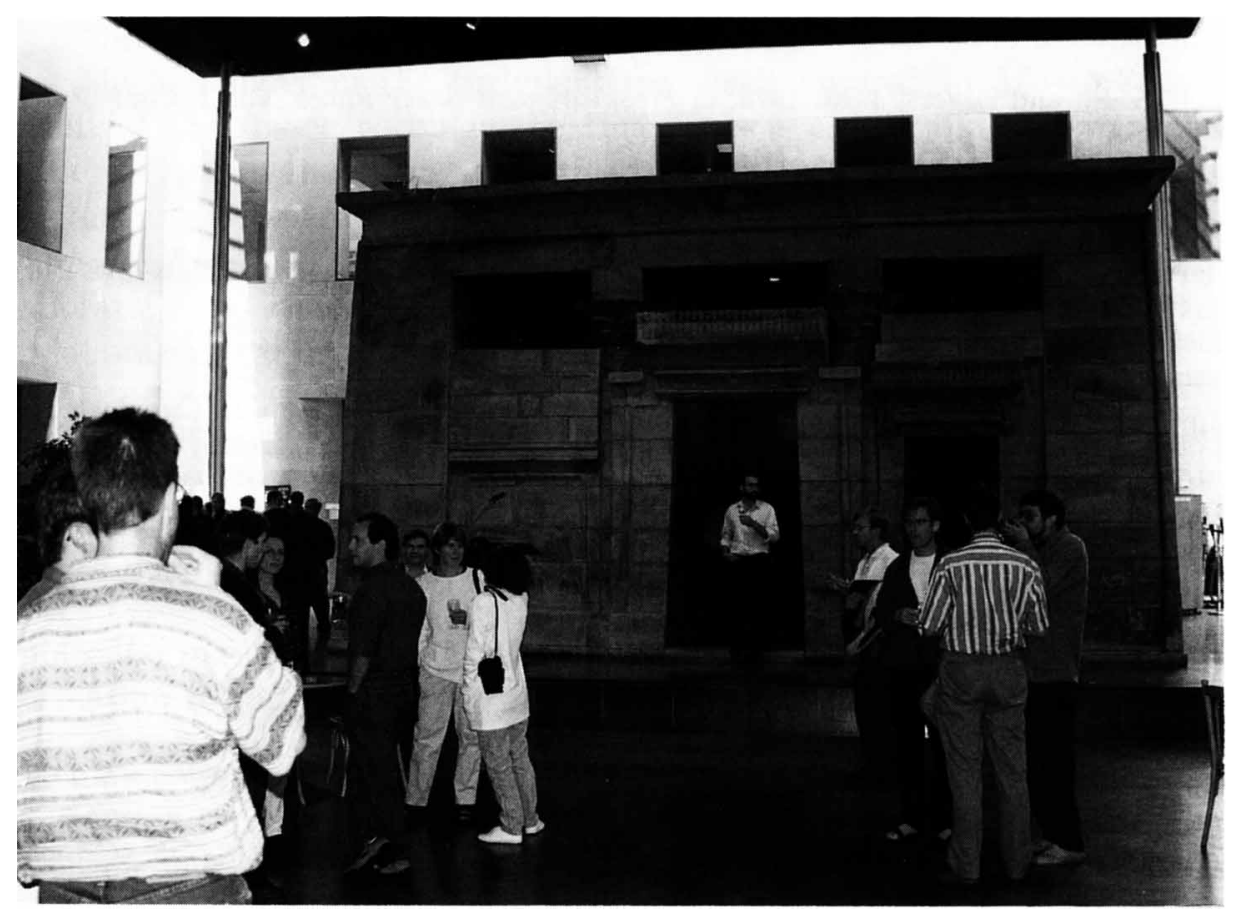

